\title{
Up-Regulation of MTHFD2 is Associated With Clinicopathological Characteistics and Poor Survival in Ovarian Cancer, Possibly By Regulating MOB1A Signaling
}

\section{Xiangrong Cui}

Children's Hospital of Shanxi

Huancheng Su

Shanxi Medical University

Jiaolin Yang

Shanxi Medical University

\section{Xueqing Wu}

Children's Hospital of Shanxi

Kai Huo

Shanxi Medical University

Xuan Jing ( $\sim$ jx05070103@163.com)

Shanxi Provincial Peoples Hospital

\section{Sanyuan Zhang}

Shanxi Medical University

\section{Research Article}

Keywords: MTHFD2, ovarian cancer, prognosis, MOB1A

Posted Date: September 29th, 2021

DOI: https://doi.org/10.21203/rs.3.rs-929562/v1

License: (a) (i) This work is licensed under a Creative Commons Attribution 4.0 International License. Read Full License 


\section{Abstract}

Background: MTHFD2 is a folate-coupled metabolic enzyme, which has been proved to participant in the metabolic reprogramming and tumor cell-sustaining proliferative capacity. However, the function of MTHFD2 in the development of ovarian cancer and its potential molecular mechanisms is still unclear.

Materials and methods: The expression, various mutations, prognosis, and related network signaling pathways of MTHFD2 were analyzed using bioinformatics-related websites, including Oncomine, GEPIA, UCSC, cBioPortal, KM Plotter, TISIDB and TIMER. The prognostic value of MTHFD2 expression was validated by our own ovarian cancer samples using RT-qPCR. The migration ad invasion of ovarian cancer cells were further analyzed by CCK-8 and transwell assay. The Western-blot assay was performed to explore the protein levels of MTHFD2 and MOB1A.

Results: We obtained the following important results. (1) MTHFD2 expression was markedly up-regulated in ovarian cancer than normal samples. (2) Among patients with ovarian cancer, those with higher MTHFD2 expression was associated with lower survival rate. (3) The major mutation type of MTHFD2 in ovarian cancer samples was missense mutation. (4) MTHFD2 knockdown inhibited proliferation, migration, invasion, as well as the expression of MOB1A in vitro.

Conclusion: MTHFD2, as a NAD+-dependent enzyme, accelerated tumor progression by up-regulating MB01A, suggesting that this protein may be an independent prognostic factor and a potential therapeutic target for future ovarian cancer treatments.

\section{Introduction}

Ovarian cancer $(\mathrm{OC})$ is recognized as the most common and lethal gynecologic malignancies, which characterized by multidrug resistance, early-onset metastasis, recurrence, and poor prognosis $[9,11,14]$. Due to a wide range of clinical, histopathological, and molecular heterogeneity, most patients were identified in the advanced stage at the time of initial diagnosis, causing an unsatisfactory 5-year survival rate of ovarian cancer patients $[11,27]$. Therefore, it is urgent to screen and identify reliable biomarkers to realize the accuracy of early diagnosis for ovarian cancer. Besides, understanding the pathophysiological mechanism of ovarian cancer may promote the optimization of treatment plan.

MTHFD2 (the mitochondrial methylenetetrahydrofolate dehydrogenase/cyclohydrolase), a folate-coupled metabolic enzyme, expressed in the developing embryo and is absent in most adult tissues $[1,10]$. Interestingly, recent studies have demonstrated that MTHFD2 confers redox homeostasis and drives cancer cell proliferation and migration [16, 20]. Markedly elevated expression of MTHFD2 was identified in many cancers and correlates with poor survival in breast cancer patients $[13,16]$. Furthermore, repression of MTHFD2 decreased the invasion and migration of breast cancer cell lines [7]. However, the exact role of MTHFD2 in ovarian cancer and its underlying regulatory mechanism have not been elucidated. 
Therefore, the current study devoted to exploring the potential value of MTHFD2 for the diagnostic and prognostic of ovarian cancer, as well as its underlying regulation mechanism. We adopt bioinformatics methods to observe the expression ad prognostic value of MTHFD2 in ovarian cancer. Besides, we used ovarian cancer cell lines to analyze the regulatory mechanism underlying the effect of highly expressed MTHFD2 on ovarian cancer. Our results demonstrated that MTHFD2 expression in ovarian cancer was up-regulated and associated with poor prognosis in ovarian cancer patients, which may play a role in malignant transformation mainly through MOB1A signaling pathway. These observations will help to develop and optimize new insights into the diagnosis and prognosis of ovarian cancer.

\section{Materials And Methods}

\section{Human sample and clinicopathological data collection}

This study was received the approval of the Ethics Committee of Shanxi Medical University (Ethical code: 2019220221). Frozen tissues specimens were collected 35 patients with ovarian cancer without any therapy before surgery at the Department of Gynaecology and Obstetrics from Tumor Hospital of Shanxi (China). The average age of the patients was 53.9 years (24-69 years). In formed written consent was obtained from all patients enrolled.

\section{Cell culture and transfections}

This human ovarian cancer cell line SKOV3 was purchased from the American Type Culture Collection (ATCC) and cells were cultured in RPMI medium 1640 (Gibco, Thermo Fisher Scientifc, Inc.) supplemented with $10 \%$ fetal bovine serum (FBS; HyClone, Logan, USA) and 1\% penicillin/streptomycin (Invitrogen, Waltham, MA) in a stable humidified atmosphere with $5 \% \mathrm{CO} 2$ at $37^{\circ} \mathrm{C}$. siRNA targeting MTHFD2 (si-MTHFD2) and siRNA negative control (si-NC) were constructed and purchased from GenePharma (Shanghai, China).

\section{Oncomine database verification}

The Oncomine (www.oncomine.org) platform is a public bioinformatics network database including 715 independent datasets and 86, 733 samples which has become an industry-standard tool cited in more than 1,100 peer-reviewed journal articles [22]. MTHFD2 gene expression levels in malignancies were analyzed, selecting the cancer type as ovarian cancer. Paired Student's t-test was performed to compare group means. A fold-change of at least 2 with a $P$-value $<0.001$ was used as clinically significant, as previously described.

\section{Gene expression profiling interactive analysis}

Gene Expression Profiling Interactive Analysis (GEPIA) database (http://gepia.cancer-pku.cn/) was performed to explore the data of tumor/normal differential expression analysis, profiling according to cancer types or pathological stages, patient survival analysis, similar gene detection, correlation analysis and dimensionality reduction analysis from TCGA and GTEx databases with bioinformatics tools 
CIBERSORT, EPIC, and quan TIseq [26]. In GEPIA, the expression of MTHFD2 and MOB1A in various human cancers and adjacent normal tissues was obtained, furthermore the expression of MTHFD2 in ovarian cancer and corresponding normal tissues was analyzed.

\section{University of California Santa Cruz (UCSC)Cancer Genomics Browser Analysis}

UCSC Xena platform (http://xenabrowseer.net), included 758 cases of ovarian cancer with genomic and clinical, was utilized to access TCGA ovarian cancer data [6]. The relationship between the mRNA expression of MTHFD2 and MOB1A was conducted thorugh TCGA-Ovarian Cancer.

\section{cBioportal database analysis}

cBioPortal (http://chioportal.org) was utilized to analyze MTHFD2 alterations observed in ovarian serous cystadenocarcinoma (TCGA, Nature 2011, $n=489$ ) $[2,5]$. By analyzing various types of mutations, putative copy number variations, and co-expression data, the tab OncoPrint displayed an overview of the genetic changes in each sample as gene mutations and heat maps of MTHFD2 expression.

\section{UALCAN analysis}

UALCAN (http://ualcan.path.uab.edu), an an open public web resource, is utilized to analyze the mRNA expression of potential genes in various tumor subtypes, including age, gender, tumor stages, and other clinicopathological features [3]. In our study, UALCAN was pertormed to access the MTHFD2 expression levels in primary ovarian carcinoma tissues and its association with multiple clinical and pathological parameters.

\section{LinkedOmics Analysis}

The LinkFinder module of LinkedOmics (http://www.linkedomics.org/login.php), included multi-omics data from all 32 TCGA Cancer types and 10 Clinical Proteomics Tumor Analysis Consortium (CPTAC) cacner cohorts, is utilized to search for attributes that are associated with a query attribute, such as mRNA or protein expression signatures of genomic alterations, candidate biomarkers of clinical attributes, and candidate target genes of transcriptional factors, microRNAs, or protein kinases [28]. In our study, LinkFinder module was performed to identify differentially expressed genes related to MTHFD2 in the TCGA OV section. To derive biological insights from the association results, the LinkInterpreter module performs enrichment analysis based on Gene Ontology, biological pathways, network modules, among other functional categories.

\section{Kaplan Meier plotter}

The Kaplan Meier plotter (http://kmplot.com/analysis/) is capable to evaluate the role of 2,190 genes on survival in ovarian cancer from the databases containing GEO, EGA, and TCGA [15]. We accessed Kaplan Meier plotter to analyze the prognostic value of MTHFD2 in ovarian cancer. The patient samples were 
split into two groups by median expression to analyze the overall survival (OS) with hazard ratios (HRs) with $95 \%$ confidence intervals and log-rank $p$-values.

\section{TIMER Database Analysis}

Tumor Immune Estimation Resource (TIMER, https://cistrome.shinyapps.io/timer/) is a website for comprehensive analysis of the tumor-infiltrating immune cells and gene expression in different types of cancers [12]. We evaluated the expression of MTHFD2 in ovarian cancer in relation to tumor purity and the abundance of immune infiltrating cells including $B$ cells, $C D 8^{+} T$ cells, $C D 4^{+} T$ cells, macrophages, neutrophils, and dendritic cells. The levels of gene expression were expressed as Log2 RSEM.

\section{TISIDB Analysis}

TISIDB database (http://cis.Hku.hk/TISIDB) is a websit for comprehensive analyzing tumor and immune cell interactions which integrates into various heterogeneous data types for each gene [23]. We access this platform to analyze the relationship between MTHDF2 expression and tumor-infiltrating lymphocytes.

\section{Quantitative RT-PCR (qRT-PCR)}

Total RNA of tissues and cells was extracted with TRIzol reagent (Invtrogen, Carlsbad, CA, USA) and the concentration of total RNA was measured with Nano-DropTM Spectrophotometers (Thermo). Appropriate amounts of total RNA were reverse transcribed using the Prime Script ${ }^{\text {TM }}$ RT Master Mix kit (Thermo Fisher Scientic, Carlsbad, CA, USA). Real-Time PCR was performed with the SYBR ${ }^{\circledR}$ Premix Ex Taq Kit (TaKaRa, Otsu, Shiga, Japan) and primers (listed below) using CFX96 (Bio-Rad company, Shanghai, China). The following PCR primer sequences as follows: MTHFD2, 5'-GATCCTGGTTGGCGAGAATCC-3' (forward) and 5'-TCTGGAAGAGG CAACTGAACA-3' (reverse), MOB1A, 5'-CAGCAGCCGCTCTTCTAAAAC-3' (forward) and 5'-CCTCAGGCAACATAACAGCTTG-3' (reverse), GAPDH, 5'-ACCACAGTCCATGCCATCA C-3' (forward); and $5^{\prime}$-TCCACCACCCTGTTGCTGTA-3' (reverse). The method of $2^{-\triangle \Delta C t}$ was performed to calculate the relative mRNA levels of target genes.

\section{Western blot}

Total protein from ovarian carcinoma cell lines was extracted using lysis buffer (KeyGEN BioTECH, China) containing phosphatase and protease inhibitors (KeyGEN BioTECH, China). Protein concentration was detected using BCA protein assay kit (KeyGEN BioTECH, China). Equal amount of protein $(10 \mu \mathrm{g})$ was loaded onto 10\% SDS-PAGE (PG112, Epizyme Biotech, China) and then transferred to PVDF membranes (Millipore, USA). All membranes were then blocked in TBST for 1 hour with $5 \%$ BSA at room temperature and followed by primary antibodies at $4{ }^{\circ} \mathrm{C}$ overnight. Then membranes were incubated at room temperature for 2 hours with HRP-conjugated secondary antibodies (701051, Zen Bioscience, China; ZB-2301, ZSGB-BIO, China). Protein signals were visualized with a chemiluminescence kit (Millipore, USA) and an Image system (Bio-Rad, USA). GAPDH was used as the internal control. Primary antibodies were 
as follows: MTHFD2 (ab151447, 1:3000, Abcam, Cambridge, MA, USA), MOB1A (ab236969, 1:3000, Abcam, Cambridge, MA, USA) and GAPDH (cat.no. T0004; 1:5000; Affinity Biosciences).

\section{CCK-8 detection of viability}

Cell Counting Kit-8 was accessed to evaluate the cell viability. Cells were seeded into 96 -well plates at $5 \times 10^{3}$ cells per well and subjected in $100 \mu \mathrm{l}$ serum-free medium for $24,48,72$, and $96 \mathrm{~h}$. Then, $10 \mu \mathrm{l} \mathrm{CCK}-8$ solution (CCK-8, ATgene, Taiwan, China) was added to each well for 2 hours and the absorbance (OD) values was detected at $450 \mathrm{~nm}$ by microplate reader (BioTek, Epoch, VT). All tests were repeated eight times, and each experiment was experimented at least three replicate.

\section{Cell migration and invasion ability assay}

Invasion and migration abilities of ovarian cancer cells transfected with si-MTHFD2 and si-NC were determined using transwell migration and invasion assay. $600 \mu \mathrm{l}$ DEME/F12 supplemented with $10 \%$ FBS was added to lower chambers, followed by Transwell chamber (Millipore, Burlington, MA, USA) seeded with $5 \times 10^{4}$ cells. For cell invasion assay, $100 \mu \mathrm{g} / \mathrm{ml}$ Matrigel (BD Biosciences, Boston, MA, USA) was added to the upper layer of chamber. After incubating for 24h, the cells in the upper chamber were removed. Cells were fixed with $600 \mu \mathrm{l}$ methanol for $10 \mathrm{~min}$. The chamber was stained with $0.25 \%$ cfystal violet (Solarbio) for $15 \mathrm{~min}$. Finally, an inverted microscope (Eclipse Ti2; Nikon Corporation) was performed to photograph and calculate the invading and migratory cells in three random fields.

\section{Statistical analysis}

GraphPad Prism 7.0 software was used to statistical analysis. Student's t-test was performed to compare the differences between two groups. One-way ANOVA was performed to compare multiple groups. Overall survival was presented as Kaplan-Meier survival curves and the statistical comparisons were calculated by Log-rank test. $P$-value $<0.05$ was set as the threshold.

\section{Results}

\section{MTHFD2 is up-regulated in human ovarian cancer}

The expression profile of MTHFD2 was identified by GEPIA. The data revealed that MTHFD2 was significantly higher in COAD, DLBC, ESCA, GBM, HNSC, LGG, LUSC, OV, READ, STAD, TGCT, THYM and lower in LAML, THCA (Fig. 1A and B). MTHFD2 transcripts were elevated significantly in ovarian cancer samples compared to normal tissue $(P<0.05)$ (Fig. 1C). To further confirmed this result, the Oncomine database was performed to analyze the expression profile of MTHFD2. Elevated mRNA expression of MTHFD2 was identified in various human tumors, including Brain and CNS Cancer, Breast Cancer, Cervical Cancer, Colorectal Cancer, Esophageal Cancer, Head and Neck Cancer, Kidndy Cancer, Leukemia, Liver Cancer, Lung Cancer, Lymphoma, Ovarian Cancer, Prostate Cancer, and Sarcoma (Fig. 2A). MTHFD2 expression was significantly elevated in ovarian mucinous adenocarcinoma, ovarian serous 
adenocarcinoma, ovarian endometrioid adenocarcinoma than in normal samples (Fig. 2B). Further subgroup analysis according multiple clinical and pathological features of ovarian carcinoma based on TCGA OV samples using UALCAN was revealed in Fig. 3. The expression level of MTHFD2 was positively associated with age and tumor grade.

\section{MTHFD2 mutations in ovarian cancer}

The pie chart in Fig. 4A generated by COSMIC summarizes the observed mutation types, including nonsense substitutions (1.97\%), missense substitutions (33.86\%), synonymous substitutions (8.66\%), inframe insertions $(0.00 \%)$, frameshift insertions $(0.79 \%)$, inframe deletions $(0.00 \%)$, frameshift deletions $(1.57 \%)$, and complex mutations $(0.00 \%)$. Furthermore, MTHFD2 mutations in ovarian cancer samples were $A>C(2.68 \%), A>G(4.46 \%), A>T(0.00 \%), C>A(13.39 \%), C>T(22.32), C>G(8.04), G>A(21.43 \%)$, $\mathrm{G}>\mathrm{C}(7.14 \%), \mathrm{G}>\mathrm{T}(8.04), \mathrm{T}>\mathrm{A}(8.04 \%), \mathrm{T}>\mathrm{C}(5.36 \%), \mathrm{T}>\mathrm{G}(2.68 \%)$. As determined using cBioPortal, the MTHFD2 mutation frequency $(0.34 \%)$ in patients with ovarian cancer. Furthermore, the additional information about all mutations (G42V and R54W) in MTHFD2 was revealed in graphical view.

\section{Relationship between MTHFD2 expression and infiltrating immune cells in ovarian cancer}

Tumor-infiltrating immune cells in the TME can independently be adopt to predict sentinel lymph node status and patient survival, and the above findings support a prognostic role of MTHFD2 in pan-cancer [4, 24]. Therefore, we used TIMER to explore the association between immune infiltration levels and MTHFD2 expression level in various cancer types. The results showed that MTHFD2 exrpession is only significantly positively correlated with neutrophils $\left(r=0.21, p=8.51 \times 10^{-4}\right)$ in ovarian cancer (Fig. $5 A$ ). Subsequently, we used TISIDB database to further explore the association between MTHFD2 expression level and 28 tumor immune infiltrating cell subtypes. These results revealed that MTHFD2 is related with 7 immune cell subtypes in ovarian cancer (Fig. 5B, Supplement 1, and Table 1). Activated CD $8 \mathrm{~T}$ cell $(r=$ $0.239, p<2.53 \mathrm{e}-05)$, activated CD4 T cell $(r=0.351, p<3.27 \mathrm{e}-10)$, effector memory CD4 T cell $(r=0.186, p$ $<0.00105)$, CD56 bright natural killer cell $(r=0.151, p<0.00831)$, CD56 dim natural killer cell $(r=0.137, p$ $<0.016)$, activated dendtritic cell $(r=0.118, p<0.0384)$, neutrophil $(r=-0.114, p<0.0468)$ are weakly correlated with MTHFD2. These results implicate that MTHFD2 plays a weak role in tumor immune infiltration regulator of ovarian cancer. 
Table 1

The correlation between MTHFD2 expression and tumor lymphocyte infiltration in ovarian cancer (TISIDB)

\begin{tabular}{|lll|}
\hline Tumor lymphocyte infiltration & $\mathbf{r}$ & $\mathbf{p}$ \\
\hline Activated CD8 T cell (Act_CD8) & 0.239 & $\mathbf{2 . 5 3 e - 0 5}$ \\
\hline Central memory CD8 T cell (Tcm_CD8) & 0.025 & 0.664 \\
\hline Effector memory CD8 T cell (Tem_CD8) & 0.039 & 0.492 \\
\hline Activated CD4 T cell (Act_CD4) & 0.351 & $\mathbf{3 . 2 7 e - 1 0}$ \\
\hline Central memory CD4 T cell (Tcm_CD4) & -0.226 & 0.654 \\
\hline Effector memory CD4 T cell (Tem_CD4) & 0.186 & 0.00105 \\
\hline T follicular helper cell (Tfh) & -0.033 & 0.564 \\
\hline Gamma delta T cell (Tgd) & 0.05 & 0.386 \\
\hline Type 1 T helper cell (Th1) & 0.004 & 0.94 \\
\hline Type 17 helper cell (Th17) & -0.039 & 0.491 \\
\hline Type 2 helper cell (Th2) & -0.014 & 0.811 \\
\hline Regulatory T cell (Treg) & 0.058 & 0.309 \\
\hline Activated B cell (Act_B) & 0.033 & 0.564 \\
\hline Immature B cell (Imm_B) & 0.043 & 0.448 \\
\hline Memory B cell (Mem_B) & -0.022 & 0.696 \\
\hline Natural killer cell (NK) & -0.012 & 0.838 \\
\hline CD56 bright natural killer cell (CD56 bright) & 0.151 & $\mathbf{0 . 0 0 8 3 1}$ \\
\hline CD56 dim natural killer cell (CD56 dim) & 0.137 & $\mathbf{0 . 0 1 6}$ \\
\hline Myeloid derived suppressor cell (MDSC) & 0.057 & 0.322 \\
\hline Natural killer T cell (NKT) & 0.051 & 0.374 \\
\hline Activated dendtritic cell (Act_DC) & 0.118 & $\mathbf{0 . 0 3 8 4}$ \\
\hline Plasmacytoid dendtritic cell (pDC) & -0.001 & 0.981 \\
\hline Immature dendtritic cell (iDC) & 0.059 & 0.3 \\
\hline Macrophage (Macrophage) & -0.025 & 0.669 \\
\hline Eosinophi (Eosinophil) & 0.438 \\
\hline Mast (Mast) & 0.775 \\
\hline
\end{tabular}




\begin{tabular}{|lll|}
\hline Tumor lymphocyte infiltration & $\mathbf{r}$ & $\mathbf{p}$ \\
\hline Monocyte (Monocyte) & 0.095 & 0.0955 \\
\hline Neutrophil (Neutrophil) & -0.114 & $\mathbf{0 . 0 4 6 8}$ \\
\hline
\end{tabular}

\section{Relationship of MTHFD2 expression and prognosis in ovarian cancer}

To evaluate whether MTHFD2 expression level has predictive significance for ovarian carcinoma prognosis, we detected the expression of MTHFD2 mRNA and its relationship with prognosis. As shown in Fig. 6A and $B$, the MTHFD2 expression was negatively related to overall survival $(H R=1.2$ [1.05-1.37], $p=0.007)$ and progression free survival $(\mathrm{HR}=1.23[1.06-1.41], p=0.0049)$. Furthermore, we detected our own clinical samples found that the overexpression of MTHFD2 mRNA and worse probabilities of survival in ovarian cancer (Fig. 6C and D).

\section{Co-expression and enrichment analysis of MTHFD2 in ovarian cancer}

To further evaluate the regulatory mechanisms underlying the role of MTHFD2 in ovarian cancer, data mining was performed on a ovarian cancer cohort using cBioPortal. Mps one binder kinase activator $1 \mathrm{~A}$ (MOB1A) is a highly related gene (Fig. 7A), which involves in the regulation of cell survival, proliferation, differentiation and organogenesis [32]. A regression analysis using cBioportal demonstrated that MTHFD2 and MOB1A levels are highly correlated (Spearman's correlation $=0.512, p$-value $=1.61 \mathrm{e}-22$ ) (Fig. 7B). The positive correction between MTHFD2 and MOB1A mRNA expression was determined using data from GEPIA (Fig. 7C). By investigating ovarian cancer data in TCGA using UCSC Xena, the positive correlation was further confirmed (Pearson's correlation $=0.6116$; Spearman's correlation $=0.6610)($ Fig. $7 D$ and E). These data informed that MTHFD2 could be associated with the MOB1A pathway in ovarian cancer. Furthermore, three independent ontologies (biological process, cellular component, and molecular function) were analyzed by gene set enrichment analysis (GSEA). The results indicated that MTHFD2 mainly involved in several biological processes (chromosome segregation, mitotic cell cycle phase transition, mitochondrial gene expression, etc.) (Fig. 8A), cellular components (mitochondrial inner membrane, condensed chromosome, mitochondrial matrix, etc.) (Fig. 8B), and molecular functions (unfolded protein binding, nucleotidytransferase activity, oxidoreductase activity, acting on $\mathrm{NAD}(\mathrm{P}) \mathrm{H}$, etc.) (Fig. 8C). Then we evaluated the potential functional pathways using KEGG (cell cycle, intestinal immune network for IgA production, human T-cell leukemia virus 1 infection, etc.) (Fig. 8D), PANTHER (ubiquitin proteasome pathway, p53 pathway, de novo pyrimidine deoxyribonucleotide biosynthesis, etc.) (Fig. 8E), and Reactome (cell cycle, DNA damage/telomere stress induced senescence, G1/S transition, etc.) (Fig. $8 \mathrm{~F})$. 


\section{MOB1A mRNA expression and prognosis in patients with ovarian cancer}

To investigate the genetic alterations of MOB1A, the GEPIA tool was performed to identify the expression profiles of MOB1A. The results of MOB1A analysis informed that MOB1A highly expressed in ovarian cancer tissues compared with matched normal tissues (Fig. 9A). Then, the prognostic value of MOB1A in ovarian cancer was identified by PrognoScan database. It was demonstrated that MOB1A mRNA expression was significantly associated with reduced overall survival (OS) time in ovarian cancer (Fig. 9B). Furthermore, our own results demonstrated the over-expression of MOB1A mRNA and worse probabilities of survival in ovarian cancer (Fig. 9C and D).

\section{MTHFD2 accelerated the biological characteristics of ovarian cancer cells through up-regulating MOB1A}

To further confirm the impact of MTHFD2 on ovarian cancer tumorigenesis, the endogenous expression level of MTHFD2 was knocked down with si-MTHFD2 transfection. As exhibited in Fig. 10A, the MTHFD2 expression was significantly reduced in ovarian cancer cells transfected with si-MTHFD2. Then, CCK-8 assay demonstrated that MTHFD2 knock-down significantly weakened the ability of proliferation of ovarian cancer (Fig. 10B). Furthermore, the transwell assay showed that knockdown of MTHFD2 obviously limited cell migration and invasion (Fig. 10C and D). Finally, the expression of MOB1A was inhibited by MTHFD2 in ovarian cancer cells (Fig. 10E and F). Above data elucidated that MTHFD2 could promot ovarian cacncer cell progression by up-regulating MOB1A in vitro.

\section{Discussion}

MTHFD2 is a NAD+-dependent enzyme with methylenetetrahydrofolate dehydrogenase and cyclohydrolase activity, which has been suggested to be a key participant in the metabolic reprogramming and tumor cell-sustaining proliferative capacity independently of dehydrogenase activity $[10,16]$. Increasing evidence suggests that MTHFD2 is overexpressed and predicts poor prognosis and promotes cancer cell proliferation and metastasis in certain types of malignancies, including colorectal cancer [29], breast cancer[8], and head and neck squamous cell cancer [8, 25]. However, the prognostic value and signal transduction of MTHFD2 expression in ovarian cancer is still ambiguous.

In the present study, to determine the role and functional network of MTHFD2 in the ovarian cancer, we performed bioinformatics analyses to analyze the extensive gene expression profiling with pre-defined parameters in ovarian cancer and normal samples. According to GEPIA database, MTHFD2 expression was significantly increased in ovarian cancer tissues compared with adjacent normal controls. The analysis of the Oncomine database revealed fold-changes in gene expression levels that MTHFD2 expression was significantly increased in ovarian mucinous adenocarcinoma, ovarian serous adenocarcinoma, ovarian endometrioid adenocarcinoma compared with normal samples. Further 
subgroup analysis according multiple clinical and pathological features of ovarian carcinoma based on TCGA OV samples using UALCAN. The expression level of MTHFD2 was positively associated with age and tumor grade. Subsequently, the mutations of MTHFD2 were identified by COSMIC and cBioPortal database. We found that the main type of mutation of MTHFD2 in ovarian cancer was missense substitutions mainly occurred in THF_DHG_CYH domain. Then, the relationship between MTHFD2 expression and infiltrating immune cells was identified by TIMER and TISIDB databse. These results suggested that MTHFD2 plays a weak role in tumor immune infiltration regulator of ovarian cancer. Prognosis analysis showed that the higher expression of MTHFD2 positively correlated with reduced OS and PFS. We further adopt our own results validated that that MTHFD2 mRNA expression may be an independent prognostic biomarker in ovarian cancer patients. Enrichment analysis of target gene sets using GSEA can identify important networks involved in protein targeting, mRNA processing, and transcription factors. MTHFD2 was showed to regulate the ubiquitin proteasome pathway, p53 pathway, de novo pyrimidine deoxyribonucleotide biosynthesis.

By mining co-expression and correlation analysis data, we determined that MTHFD2 and MOB1A were both upregulated in ovarian cancer. Mps One Binder Kinase Activator (MOB)1A, one of the most core components of the Hippo pathway, was considered to influence biological functions in various cancers $[17,18,31]$. Furthermore, multiple studies have revealed that MOB1A is involved in development and progress in many types of cancers, such as glioma [30], gallbladder carcinoma [32], cervical carcinoma [19], pancreatic ductal adenocarcinoma [21], and gastric cancer [31]. Then, we identified the expression of MOB1A in ovarian cancer through GEPIA database and our own samples. The results demonstrated that MOB1A mRNA expression was significantly up-regulated and correlated with poor survival in ovarian carcinoma samples compared with controls. These results illustrated that the expression of MOB1A may regulate tumor invasion and survival correlated with MOB1A.

Subsequently, we further identified the function of MTHFD2 in growth and metastasis of ovarian cancer cells through knockdown the expression of MTHFD2 using siRNA technology. Our experiments confirmed that MTHFD2 might be a potential factor promoting the proliferation, migration, and invasion of ovarian cancer. Consistently, after knock down MTHFD2, we found that the expression of MOB1A was significantly decreased in ovarian cancer cell lines. These experiments preliminarily demonstrate that MTHFD2 exerted its effects on the malignant behaviors of ovarian cancer cells through the MOB1A signaling pathway.

\section{Conclusion}

In summary, through our study found that MTHFD2 is highly expressed in ovarian cancer, as well as an indispensable risk factor for poor prognosis in patients with ovarian cancer. We also found that that MTHFD2 has a role in malignancy mainly through the MOB1A signaling, which is a potential target for treating ovarian cancer. However, some limitations remain as the potential regulatory mechanism of MTHFD2 in ovarian were not verified sufficiently and required further exploration. 


\section{Declarations}

Acknowledgements

Not applicable.

\section{Author's contributions}

All authors contributed to data analysis, drafting or revising the article, gave final approval of the version to be published, and agree to be accountable for all aspects of the work.

\section{Ethics Approval and Consent to Participate}

Informed consent was obtained from each participant. The entire experiments have already obtained the approval of the Ethics Committee of Shanxi Medical University (Ethical code: 201922021)

\section{Funding}

This study was supported by the Scientifc Research Project of Shanxi Provincial Department of Health (grant no. 201601070), Initial Scientifc Research Fund of PhD in Shanxi Provincial People's Hospital (grant no. b201635), Natural Science Foundation of Shanxi (grant no. 201901D211519 and 201901D211546), Research Project Supported by Shanxi Scholarship Council of China (grant no. HGKY2019092), and China Postdoctoral Science Foundation (grant no. 2020M670703), Fund Program for the Scientific Activities of Selected Returned Overseas Professionals in Shanxi Province (grant no. 20200033), National Natural Science Foundation of China (grant no. 82000722 and 82000302) , Medical and Technological Innovation Team of Shanxi (2020TD19).

\section{Availability of data and materials}

The analyzed data sets generated during the study are available from the corresponding author on reasonable request.

\section{Consent for publication}

Not applicable.

\section{Competing interests}

The authors declare that they have no competing interests.

Author details

${ }^{1}$ Reproductive Medicine Center, The affiliated Children's Hospital of Shanxi Medical University, Children's Hospital of Shanxi, Women Health Center of Shanxi, Taiyuan, 030001 (China); ${ }^{2}$ Gynaecology and Obstetrics Department, First Hospital of Shanxi Medical University, Taiyuan, 030001 (China); ${ }^{3}$ Clinical 
Laboratory, The Affiliated People's Hospital of Shanxi Medical University, Taiyuan, China., 030001 (China);

${ }^{4}$ Breast Surgery Department, Tumor Hospital of Shanxi, Affiliated of Shanxi Medical University, Taiyuan, 030000 (China)

\section{References}

1. Ben-Sahra I, Hoxhaj G, Ricoult SJH, Asara JM, Manning BD. mTORC1 induces purine synthesis through control of the mitochondrial tetrahydrofolate cycle. Science. 2016;351(6274):728-33.

2. Cao Q, Song Z, Ruan H, Wang C, Yang X, Bao L, Wang K, Cheng G, Xu T, Xiao W, et al. Targeting the KIF4A/AR Axis to Reverse Endocrine Therapy Resistance in Castration-resistant Prostate Cancer. Clinical cancer research: an official journal of the American Association for Cancer Research. 2020;26(6):1516-28.

3. Chandrashekar DS, Bashel B, Balasubramanya SAH, Creighton CJ, Ponce-Rodriguez I, Chakravarthi B, Varambally S. UALCAN: A Portal for Facilitating Tumor Subgroup Gene Expression and Survival Analyses. Neoplasia. 2017;19(8):649-58.

4. Cui L, Chen $\mathrm{H}$, Zhao X. The Prognostic Significance of Immune-Related Metabolic Enzyme MTHFD2 in Head and Neck Squamous Cell Carcinoma. Diagnostics (Basel) 2020, 10(9).

5. Gao J, Aksoy BA, Dogrusoz U, Dresdner G, Gross B, Sumer SO, Sun Y, Jacobsen A, Sinha R, Larsson E, et al. Integrative analysis of complex cancer genomics and clinical profiles using the cBioPortal. Sci Signal. 2013;6(269):pl1.

6. Goldman MJ, Craft B, Hastie M, Repecka K, McDade F, Kamath A, Banerjee A, Luo Y, Rogers D, Brooks AN, et al. Visualizing and interpreting cancer genomics data via the Xena platform. Nat Biotechnol. 2020;38(6):675-8.

7. Harlid S, Ivarsson MI, Butt S, Hussain S, Grzybowska E, Eyfjord JE, Lenner P, Forsti A, Hemminki K, Manjer J, et al: A candidate CpG SNP approach identifies a breast cancer associated ESR1-SNP. International journal of cancer 2011, 129(7):1689-1698.

8. Huang J, Qin Y, Lin C, Huang X, Zhang F. MTHFD2 facilitates breast cancer cell proliferation via the AKT signaling pathway. Experimental therapeutic medicine. 2021;22(1):703.

9. Hurwitz LM, Pinsky PF, Trabert B. General population screening for ovarian cancer. Lancet. 2021;397(10290):2128-30.

10. Ju HQ, Lu YX, Chen DL, Zuo ZX, Liu ZX, Wu QN, Mo HY, Wang ZX, Wang DS, Pu HY, et al. Modulation of Redox Homeostasis by Inhibition of MTHFD2 in Colorectal Cancer: Mechanisms and Therapeutic Implications. J Natl Cancer Inst. 2019;111(6):584-96.

11. Kuroki L, Guntupalli SR. Treatment of epithelial ovarian cancer. Bmj. 2020;371:m3773.

12. Li T, Fan J, Wang B, Traugh N, Chen Q, Liu JS, Li B, Liu XS. TIMER: A Web Server for Comprehensive Analysis of Tumor-Infiltrating Immune Cells. Cancer research. 2017;77(21):e108-10.

13. Liu F, Liu Y, He C, Tao L, He X, Song H, Zhang G. Increased MTHFD2 expression is associated with poor prognosis in breast cancer. Tumour biology: the journal of the International Society for 
Oncodevelopmental Biology Medicine. 2014;35(9):8685-90.

14. Menon U, Gentry-Maharaj A, Burnell M, Singh N, Ryan A, Karpinskyj C, Carlino G, Taylor J, Massingham SK, Raikou M, et al. Ovarian cancer population screening and mortality after long-term follow-up in the UK Collaborative Trial of Ovarian Cancer Screening (UKCTOCS): a randomised controlled trial. Lancet. 2021;397(10290):2182-93.

15. Nagy A, Lanczky A, Menyhart O, Gyorffy B. Author Correction: Validation of miRNA prognostic power in hepatocellular carcinoma using expression data of independent datasets. Scientific reports. 2018;8(1):11515.

16. Nilsson R, Jain M, Madhusudhan N, Sheppard NG, Strittmatter L, Kampf C, Huang J, Asplund A, Mootha VK. Metabolic enzyme expression highlights a key role for MTHFD2 and the mitochondrial folate pathway in cancer. Nature communications. 2014;5:3128.

17. Nishio M, Hamada K, Kawahara K, Sasaki M, Noguchi F, Chiba S, Mizuno K, Suzuki SO, Dong Y, Tokuda M, et al. Cancer susceptibility and embryonic lethality in Mob1a/1b double-mutant mice. J Clin Investig. 2012;122(12):4505-18.

18. Nishio M, Sugimachi K, Goto H, Wang J, Morikawa T, Miyachi Y, Takano Y, Hikasa H, Itoh T, Suzuki SO, et al: Dysregulated YAP1/TAZ and TGF-beta signaling mediate hepatocarcinogenesis in Mob1a/1b-deficient mice. Proceedings of the National Academy of Sciences of the United States of America 2016, 113(1):E71-80.

19. Nishio M, To Y, Maehama T, Aono Y, Otani J, Hikasa H, Kitagawa A, Mimori K, Sasaki T, Nishina H, et al. Endogenous YAP1 activation drives immediate onset of cervical carcinoma in situ in mice. Cancer Sci. 2020;111(10):3576-87.

20. Pikman Y, Puissant A, Alexe G, Furman A, Chen LM, Frumm SM, Ross L, Fenouille N, Bassil CF, Lewis CA, et al. Targeting MTHFD2 in acute myeloid leukemia. J Exp Med. 2016;213(7):1285-306.

21. Quan M, Chen Z, Jiao F, Xiao X, Xia Q, Chen J, Chao Q, Li Y, Gao Y, Yang H, et al. Lysine demethylase 2 (KDM2B) regulates hippo pathway via MOB1 to promote pancreatic ductal adenocarcinoma (PDAC) progression. Journal of experimental clinical cancer research: CR. 2020;39(1):13.

22. Rhodes DR, Yu J, Shanker K, Deshpande N, Varambally R, Ghosh D, Barrette T, Pandey A, Chinnaiyan AM. ONCOMINE: a cancer microarray database and integrated data-mining platform. Neoplasia. 2004;6(1):1-6.

23. Ru B, Wong CN, Tong Y, Zhong JY, Zhong SSW, Wu WC, Chu KC, Wong CY, Lau CY, Chen I, et al. TISIDB: an integrated repository portal for tumor-immune system interactions. Bioinformatics. 2019;35(20):4200-2.

24. Shang M, Yang H, Yang R, Chen T, Fu Y, Li Y, Fang X, Zhang K, Zhang J, Li H, et al. The folate cycle enzyme MTHFD2 induces cancer immune evasion through PD-L1 up-regulation. Nature communications. 2021;12(1):1940.

25. Shukla K, Singh N, Lewis JE, Tsang AW, Boothman DA, Kemp ML, Furdui CM. MTHFD2 Blockade Enhances the Efficacy of beta-Lapachone Chemotherapy With lonizing Radiation in Head and Neck Squamous Cell Cancer. Front Oncol. 2020;10:536377. 
26. Tang Z, Kang B, Li C, Chen T, Zhang Z. GEPIA2: an enhanced web server for large-scale expression profiling and interactive analysis. Nucleic acids research. 2019;47(W1):W556-60.

27. Tew WP, Lacchetti C, Ellis A, Maxian K, Banerjee S, Bookman M, Jones MB, Lee JM, Lheureux S, Liu $\mathrm{JF}$, et al. PARP Inhibitors in the Management of Ovarian Cancer: ASCO Guideline. Journal of clinical oncology: official journal of the American Society of Clinical Oncology. 2020;38(30):3468-93.

28. Vasaikar SV, Straub P, Wang J, Zhang B. LinkedOmics: analyzing multi-omics data within and across 32 cancer types. Nucleic acids research. 2018;46(D1):D956-63.

29. Wan X, Wang C, Huang Z, Zhou D, Xiang S, Qi Q, Chen X, Arbely E, Liu CY, Du P, et al. Cisplatin inhibits SIRT3-deacetylation MTHFD2 to disturb cellular redox balance in colorectal cancer cell. Cell death disease. 2020;11(8):649.

30. Wang J, Liu B, Yao J, Liu Z, Wang H, Zhang B, Lian X, Ren Z, Liu L, Gao Y. Interleukin-1 receptorassociated kinase 4 as a potential biomarker: Overexpression predicts poor prognosis in patients with glioma. Oncology letters. 2021;21(4):254.

31. Wang L, Li B, Zhang L, Li Q, He Z, Zhang X, Huang X, Xu Z, Xia Y, Zhang Q, et al. miR-664a-3p functions as an oncogene by targeting Hippo pathway in the development of gastric cancer. Cell proliferation. 2019;52(3):e12567.

32. Yang B, Li Y, Zhang R, Liu L, Miao H, Li Y, Shao Z, Ren T, Zhang Y, Zhang Q, et al. MOB1A regulates glucose deprivation-induced autophagy via IL6-STAT3 pathway in gallbladder carcinoma. American journal of cancer research. 2020;10(11):3896-910.

\section{Figures}


A MTHFD2 median expression of tumor and normal samples in bodymap

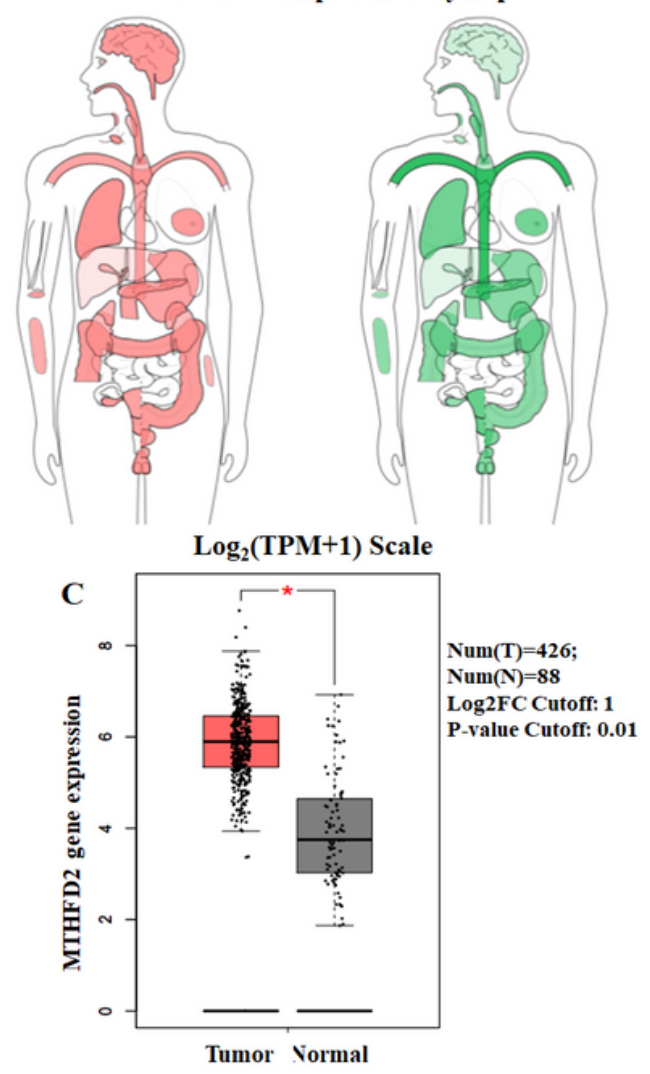

B MTHFD2 expression profile across all tumor samples and paired normal tissues.(Dot plot) Each dots represent expression of samples.

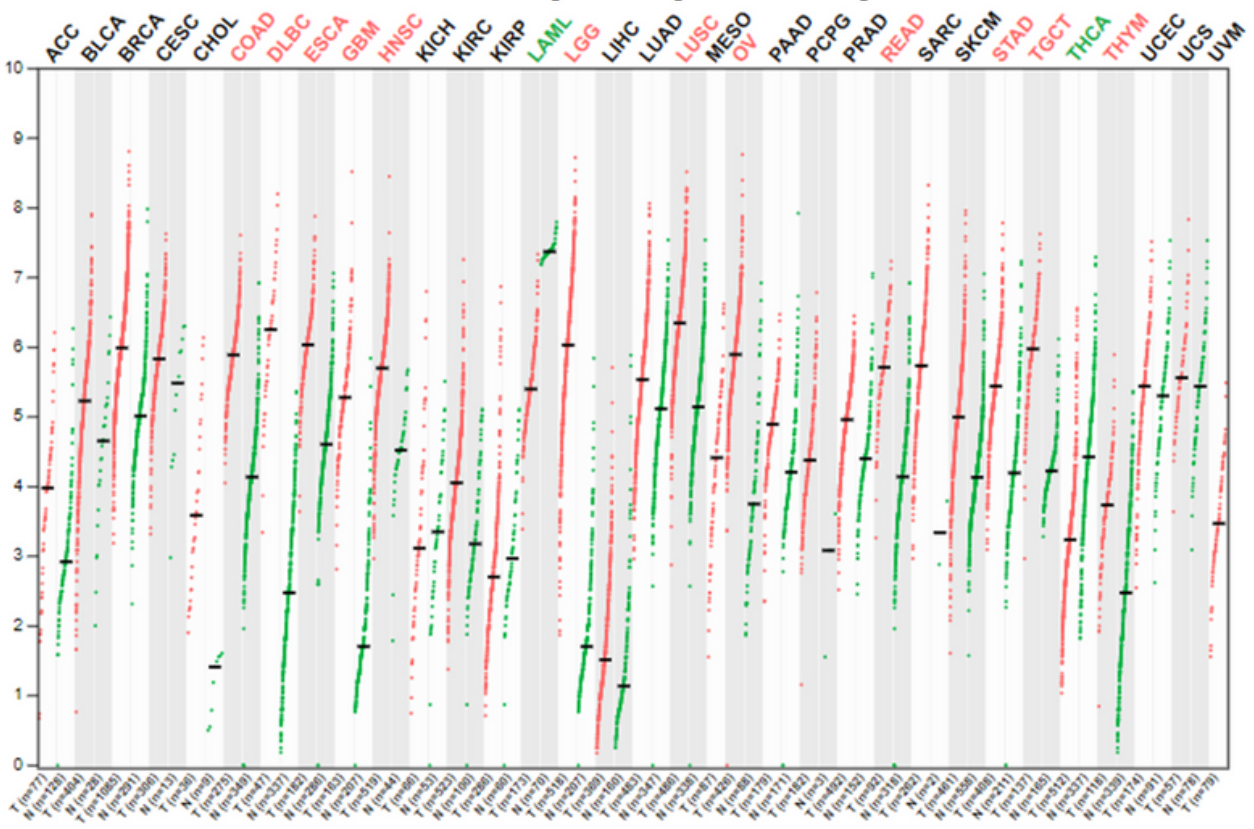

Figure 1

Expression of MTHFD2 in ovarian cancer and normal tissues from GEPIA databases. A: MTHFD2 median expression of tumor (red) and normal (green) samples in body map. B: MTHFD2 expression profile across all tumor (red) and paired normal (green) tissues. Each dot represents the expression of sample. C: The expression of MTHFD2 mRNA in ovarian cancer tissues (red box) and paired normal tissues (black box) from GEPIA. 


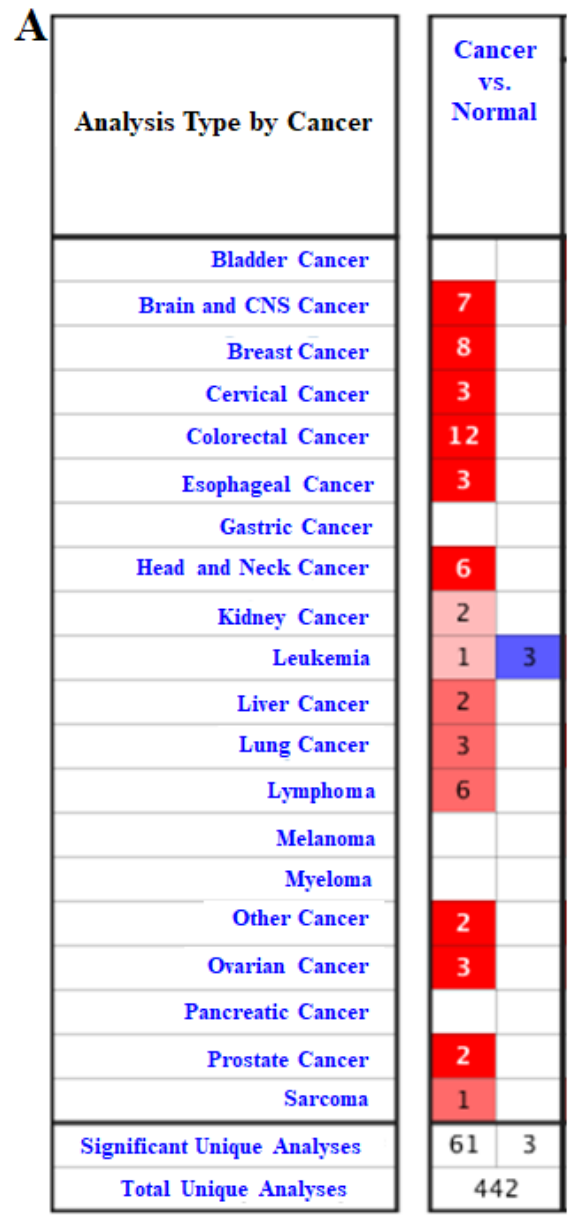

$\mathbf{B}$
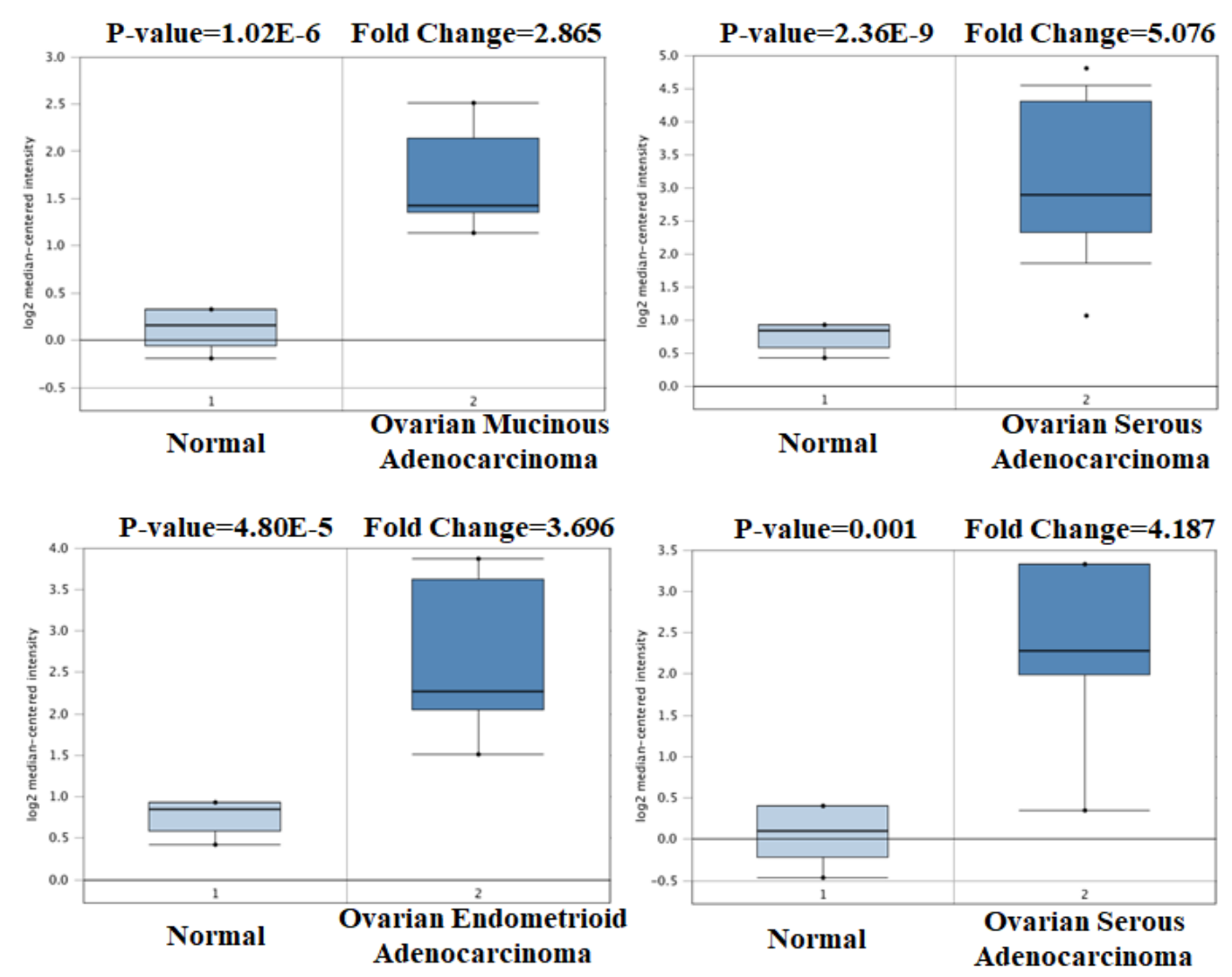

\section{Figure 2}

MTHFD2 analysis in ovarian cancer (Oncomine database). A: Expression of MTHFD2 in different tumors. Graphs show the number of datasets with statistically significant mRNA overexpression (red) or downregulation (blue) of MTHFD2. B: Box plots derived from gene expression data in Oncomine comparing the expression of MTHFD2 in normal and ovarian cancer tissue. 
A Expression of MTHFD2 in Ovarian Cancer based on patient's race

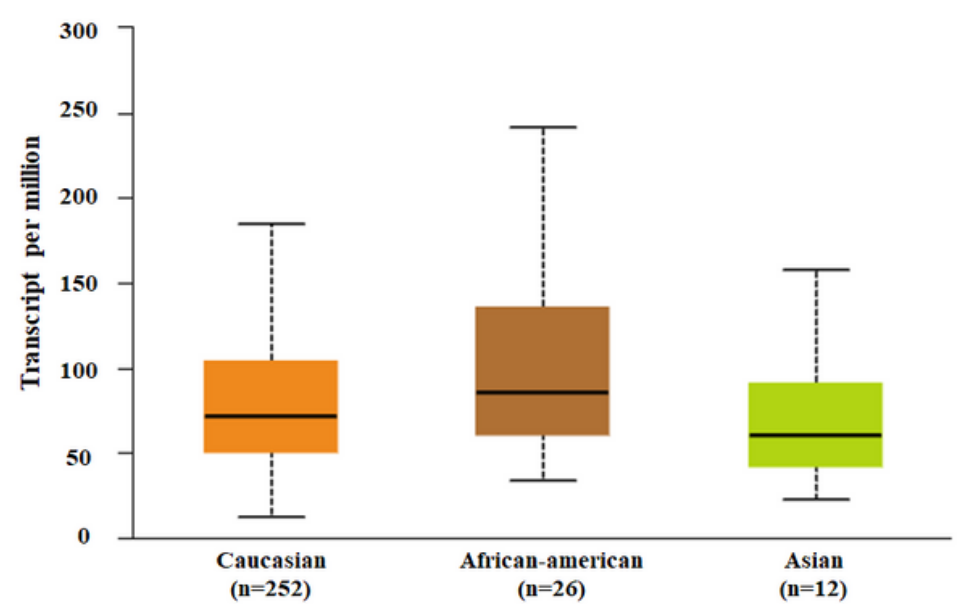

C Expression of MTHFD2 in Ovarian Cancer based on TP53 mutation status

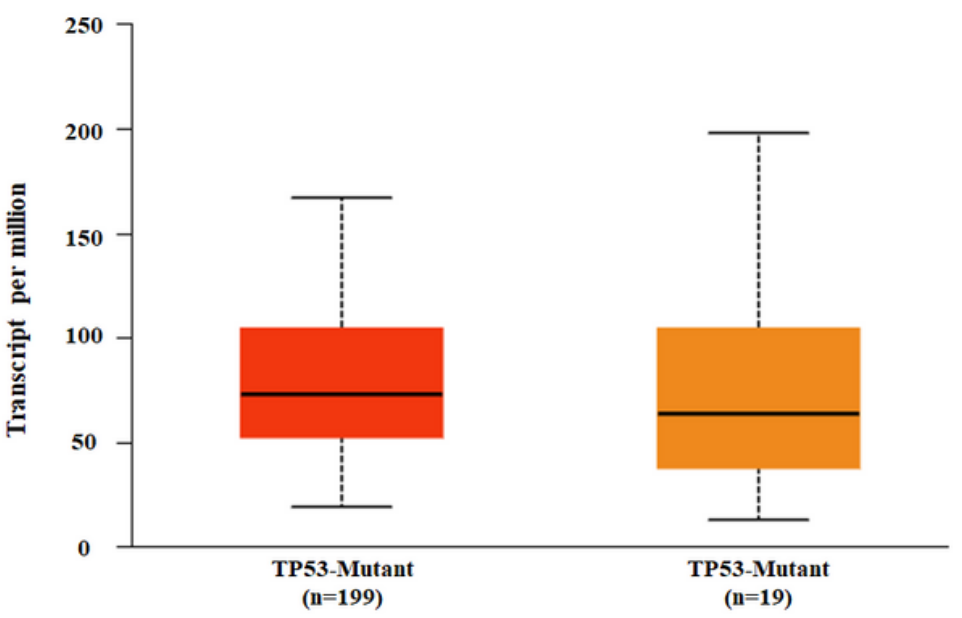

B

Expression of MTHFD2 in Ovarian Cancer based on patient's age

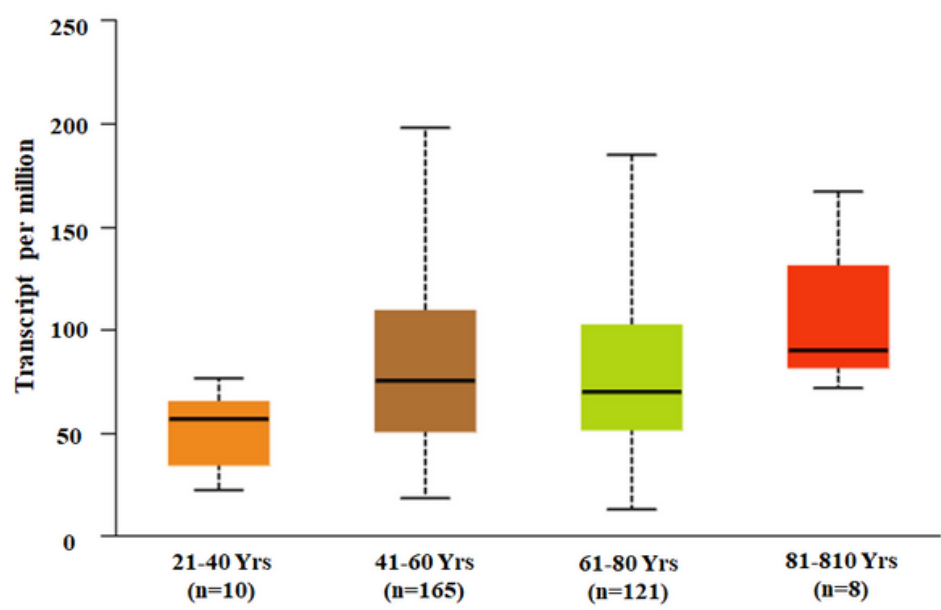

D Expression of MTHFD2 in Ovarian Cancer based on tumor grade

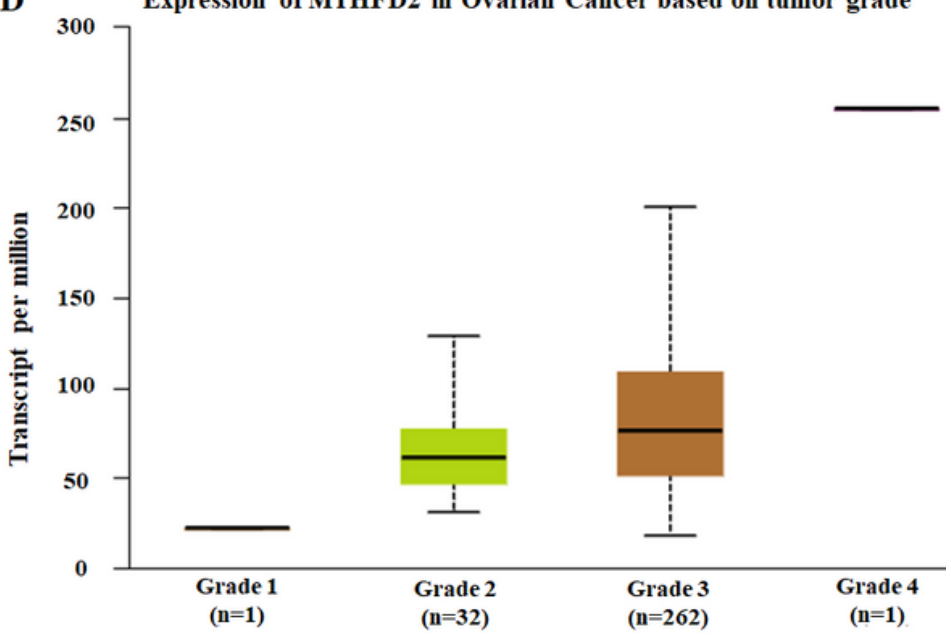

\section{Figure 3}

Genetic alterations of MTHFD2 in ovarian cancer subgroups. Subgroup analysis of multiple clinic pathologic features of ovarian cancer samples in the TCGA. A: rases; B: Ages; C: TP53-Mutant; D: stages. 
Nonsense substitution (\%)

Missense substitution

$5(1.97 \%)$

Synonymous substitution $22(8.66 \%)$

Inframe insertion

$0(0.00 \%)$

Frameshift insertion

$2(0.79 \%)$

Inframe deletion

$0(0.00 \%)$

Frameshift deletion

$4(1.57 \%)$

Complex mutation

$0(0.00 \%)$

Other

$30(11.81 \%)$

Total unique samples

254

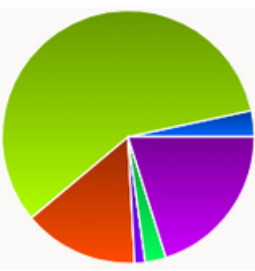

\begin{tabular}{|c|c|c|}
\hline Colour & $\begin{array}{l}\text { Mutation } \\
\text { type }\end{array}$ & $\begin{array}{l}\text { Number of } \\
\text { samples }(\%)\end{array}$ \\
\hline & $A>C$ & $3(2.68 \%)$ \\
\hline & $\underline{A}>\mathrm{G}$ & $5(4.46 \%)$ \\
\hline & $A>T$ & $0(0.00 \%)$ \\
\hline & $C>A$ & $15(13.39 \%)$ \\
\hline & $C>T$ & $25(22.32 \%)$ \\
\hline & $\underline{C>G}$ & $9(8.04 \%)$ \\
\hline & $\mathrm{G}>\mathrm{A}$ & $24(21.43 \%)$ \\
\hline & $\underline{G}>C$ & $8(7.14 \%)$ \\
\hline & $\mathrm{G}>\mathrm{T}$ & $9(8.04 \%)$ \\
\hline & $\underline{T}>\mathrm{A}$ & $9(8.04 \%)$ \\
\hline & $T>C$ & $6(5.36 \%)$ \\
\hline & $\underline{T>G}$ & $3(2.68 \%)$ \\
\hline
\end{tabular}

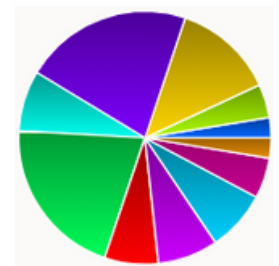

B

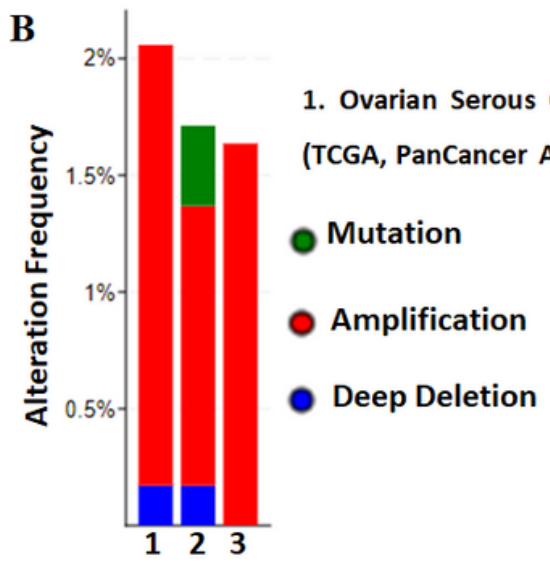

Total unique samples 112

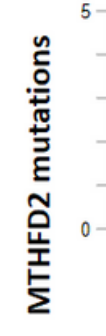

\section{Figure 4}

MTHFD2 mutations in human ovarian cancer. A: The pie chart generated by COSMIC summarizes the observed mutation types, including nonsense substitutions, missense substitutions, synonymous substitutions, inframe insertions, frameshift insertions, inframe deletions, frameshift deletions, and complex mutations. B: As determined using cBioPortal, the MTHFD2 mutation frequency $(0.34 \%)$ in patients with ovarian cancer. 

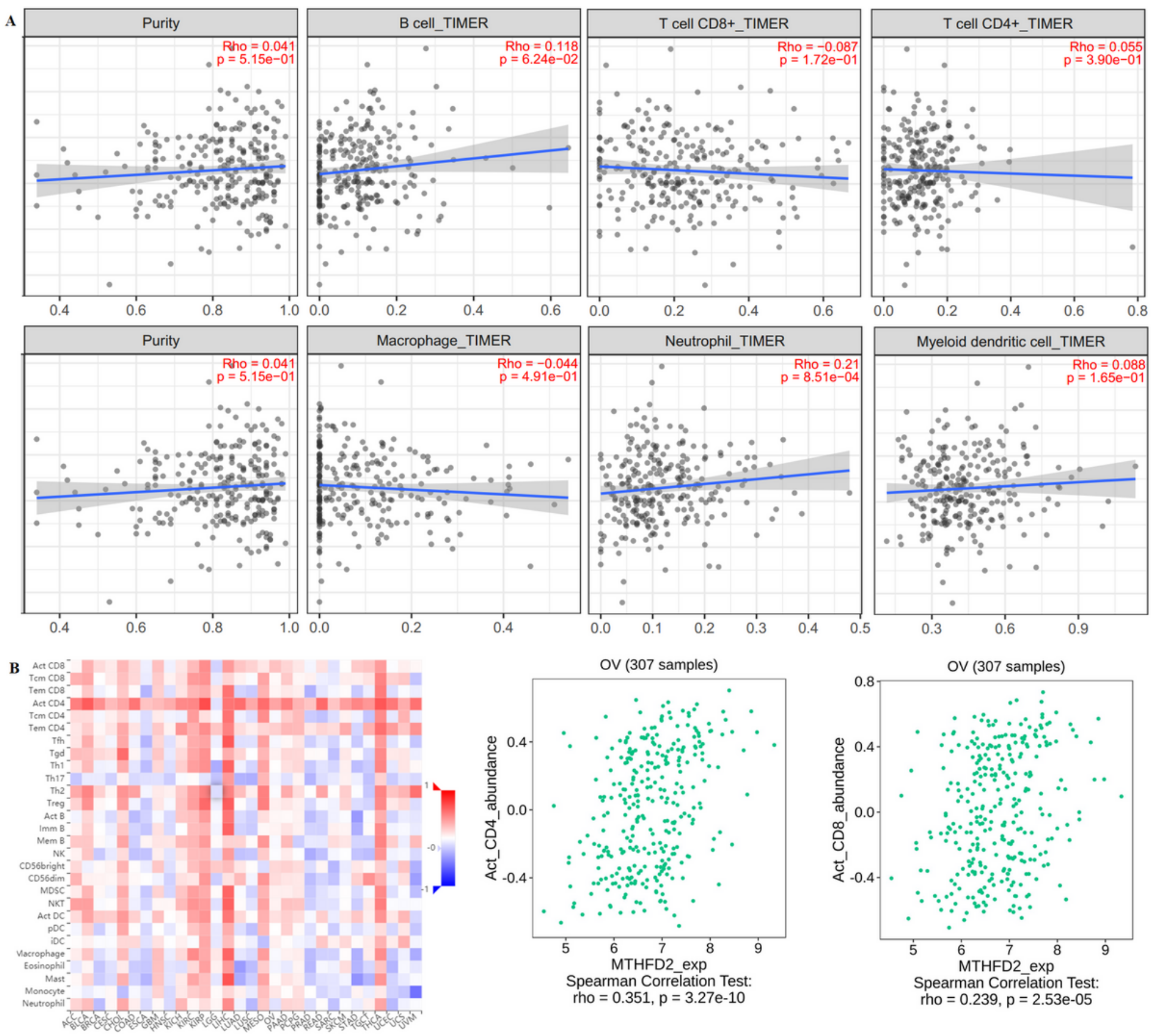

Figure 5

Correlation analysis of MTHFD2 level an immune cells infiltration levels across human cancers using the TIMER dataset and TISIDB database. A: MTHFD2 exrpession is only significantly positively correlated with neutrophils in ovarian cancer. B: MTHFD2 is related with 7 immune cell subtypes in ovarian cancer. 
A

MTHFD2 (201761_at)

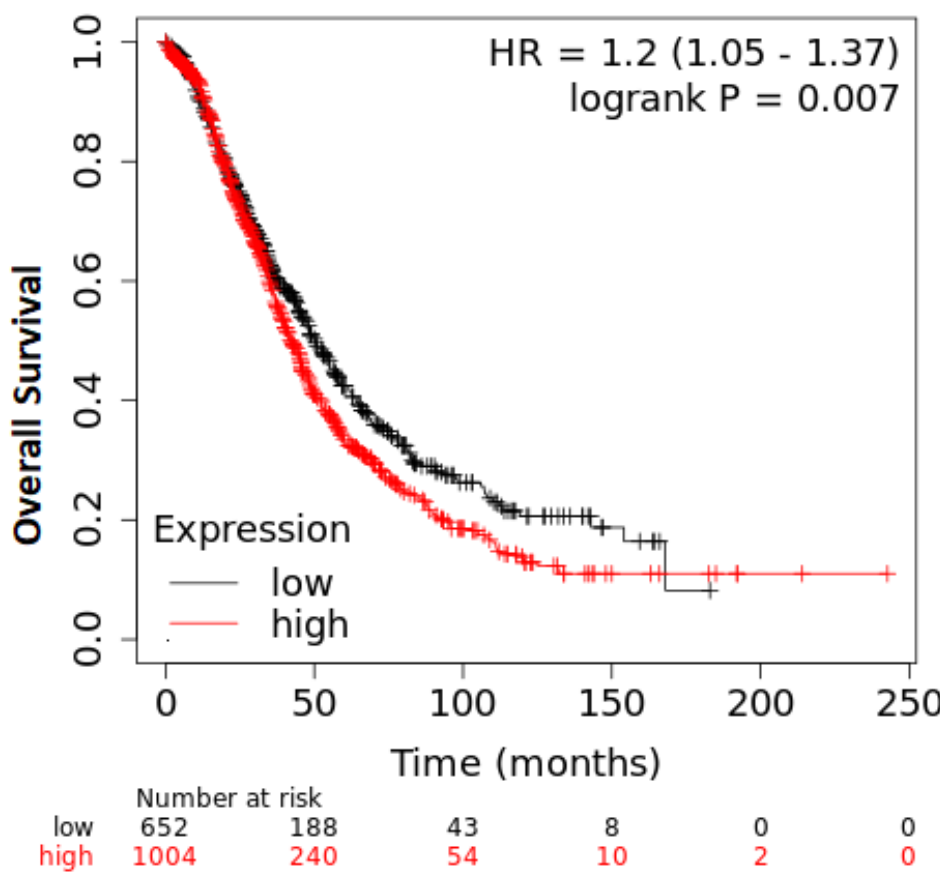

\section{C}

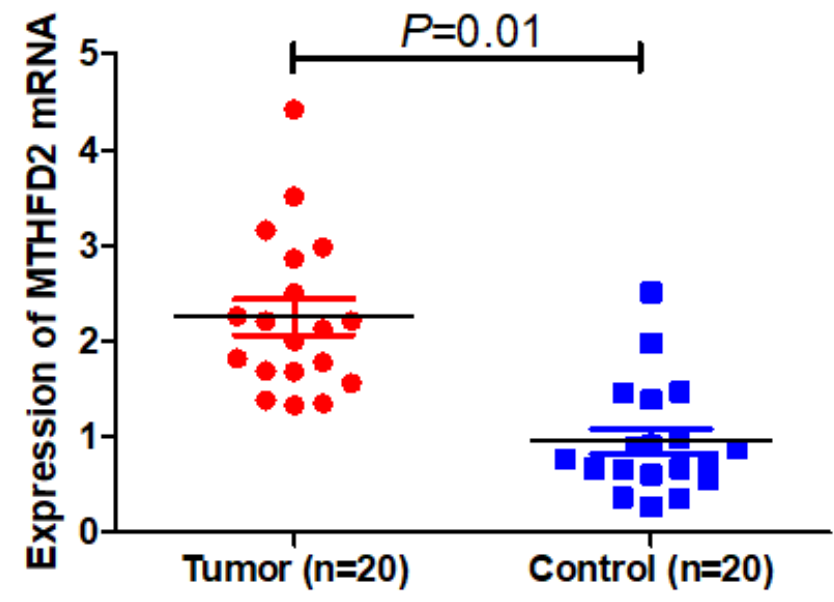

B MTHFD2 (201761_at)

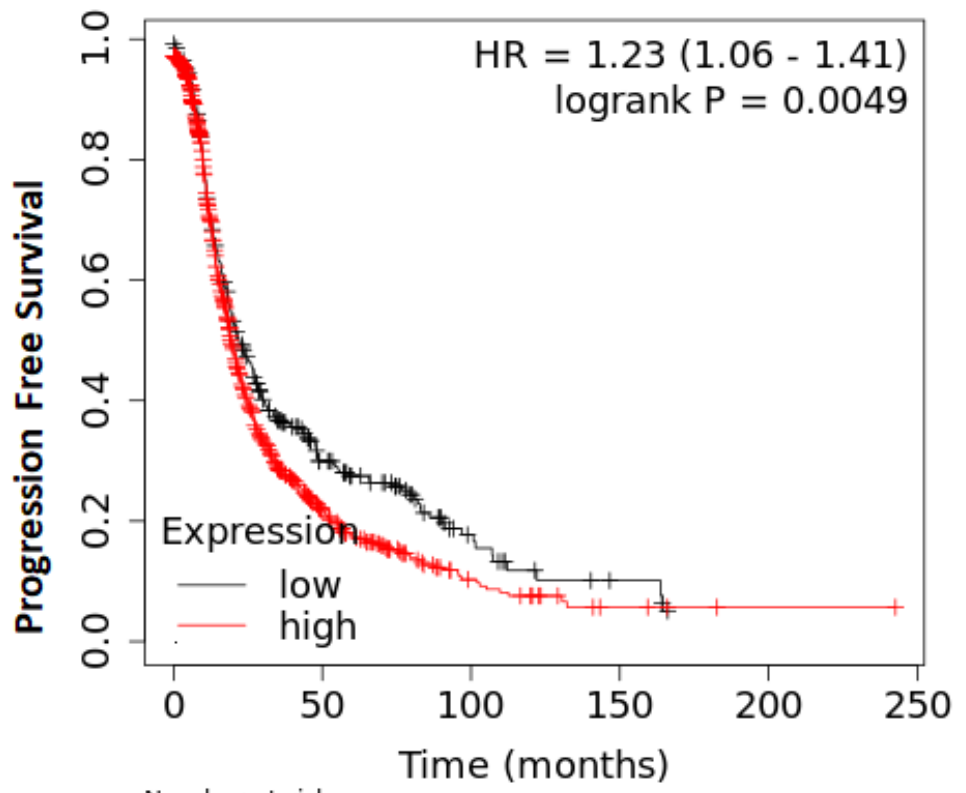

\begin{tabular}{rcccccc}
\multicolumn{7}{c}{ Number at risk } \\
low & 374 & 65 & 16 & 4 & 0 & 0 \\
high & 1061 & 107 & 19 & 4 & 1 & 0
\end{tabular}

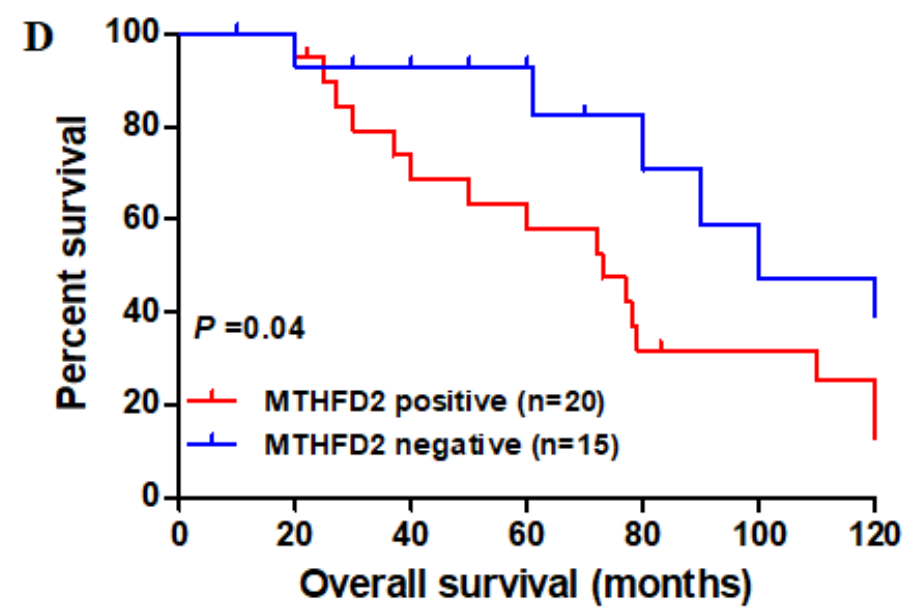

Figure 6

MTHFD2 as a prognosis marker in ovarian cancer. A: Overall survival (OS) curves calculated by KaplanMeier Plotter Analysis. B: Progression Free Survival (PFS) curves calculated by Kaplan-Meier Plotter Analysis. C: Expression of MTHFD2 in tumor (20 cases) and adjacent normal tissues (20 cases). D: Kaplan-Meier curves based on MTHFD2 expression were drawn for overall survival in 35 patients. 
A Top15 genes positively correlated to MTHFD2 expression correlated Gene cytoband $\begin{gathered}\text { Spearman's } \\ \text { Correlation }\end{gathered}$-Value q-Value $\begin{array}{lrrrr}\text { MOB1A } & 2 \mathrm{p} 13.1 & 0.512 & 1.61 \mathrm{e}-22 & \mathbf{2 . 9 6 e - 1 8}\end{array}$ $\begin{array}{lllll}\text { CCNA2 } & 4 \mathrm{q} 27 & 0.427 & 2.01 \mathrm{e}-15 & 1.85 \mathrm{e}-11\end{array}$ $\begin{array}{lllll}\text { MRPL19 } & 2 \mathrm{p} 12 & 0.411 & 2.54 \mathrm{e}-14 & 1.56 \mathrm{e}-10\end{array}$ $\begin{array}{lllll}\text { ALDH1L2 } & 12 \mathrm{q} 23.3 & 0.406 & 5.34 \mathrm{e}-14 & \mathbf{2 0 4 \mathrm { e }} \cdot 10\end{array}$ PSME4 2p16.2

PLK4 $4 \mathrm{q} 28.1$

PNPT1 2p16.1

CXCR4 2 q22.1

CDK1 $10 \mathrm{q} 21.2$

ABCE1 $4 \mathrm{q} 31.21$

XPOT $12 \mathrm{q} 14.2$

PEX13 2p15

VPS54 2p15-p14

PPA1 $10 \mathrm{q} 22.1$

MRPL35 2 P11.2

D

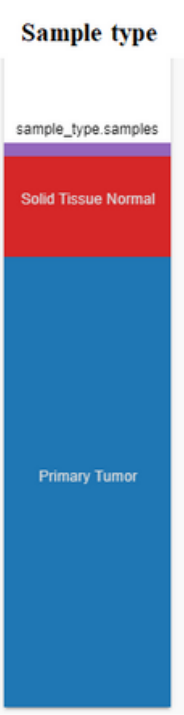

\section{MTHFD2} expression expression
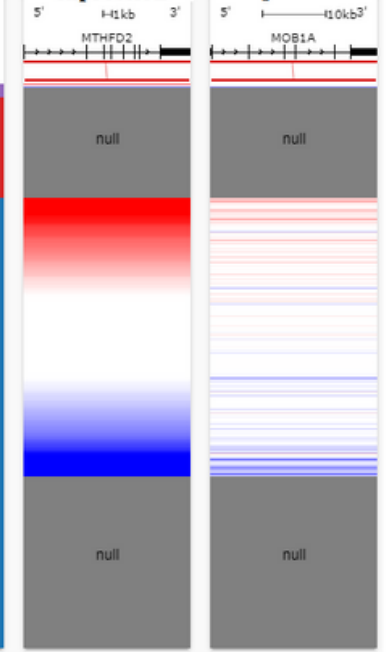

$\square$ Recurrent Tumor

$\square$ Solid Tissue No...

$\square$ Primary Tumor
B

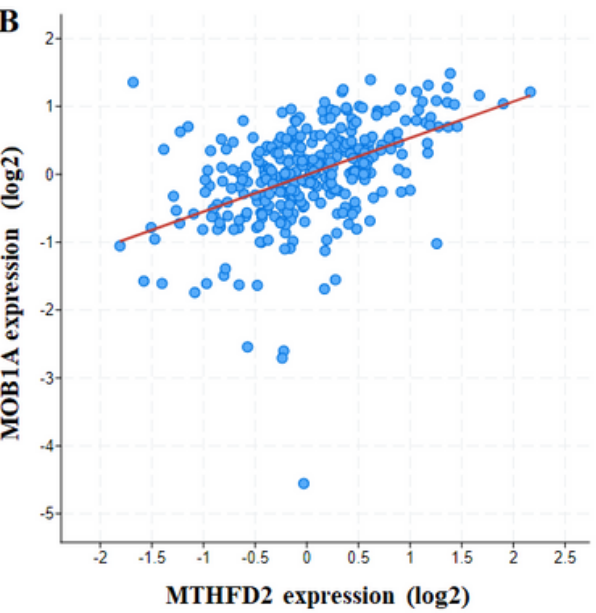

E

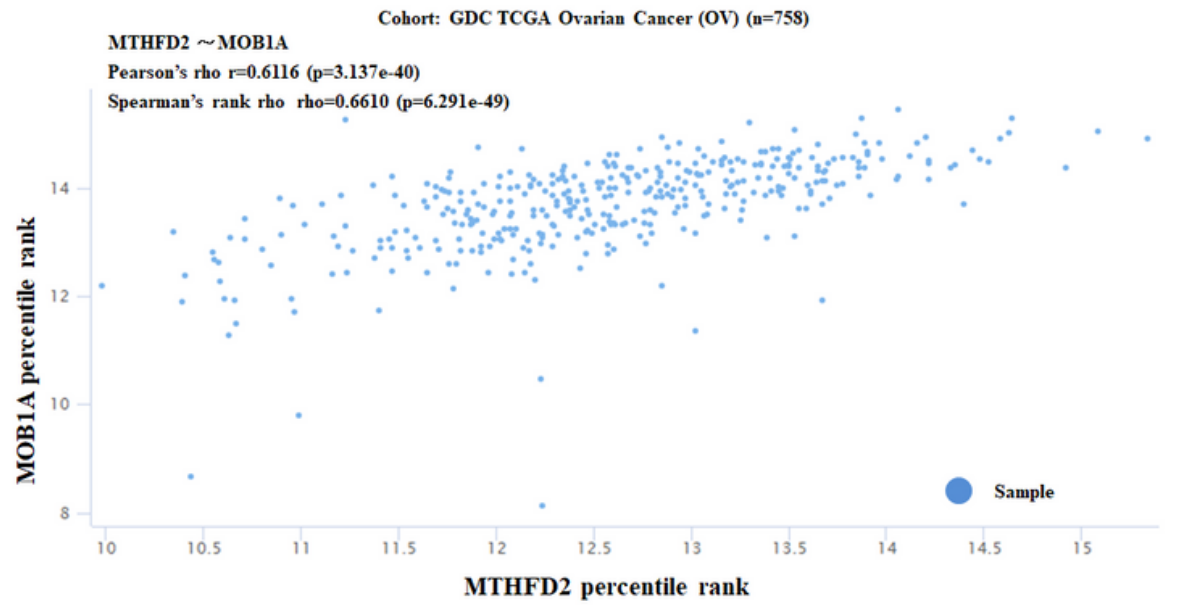

Figure 7

Co-Expression of MTHFD2 gene. A: Co-expression of MTHFD2 gene as detected by cBioPortal. B: Regression analysis between MTHFD2 and MOB1A in ovarian cancer determined by cBioPortal. C: Relationship between MTHFD2 and MOB1A in ovarian cancer by GEPIA. D खHeat map of MTHFD2 and MOB1A mRNA expression in ovarian cancer identified by UCSC Xena. E: Correlation between MTHFD2 and MOB1A mRNA expression in the TCGA database, identified by UCSC Xena. 

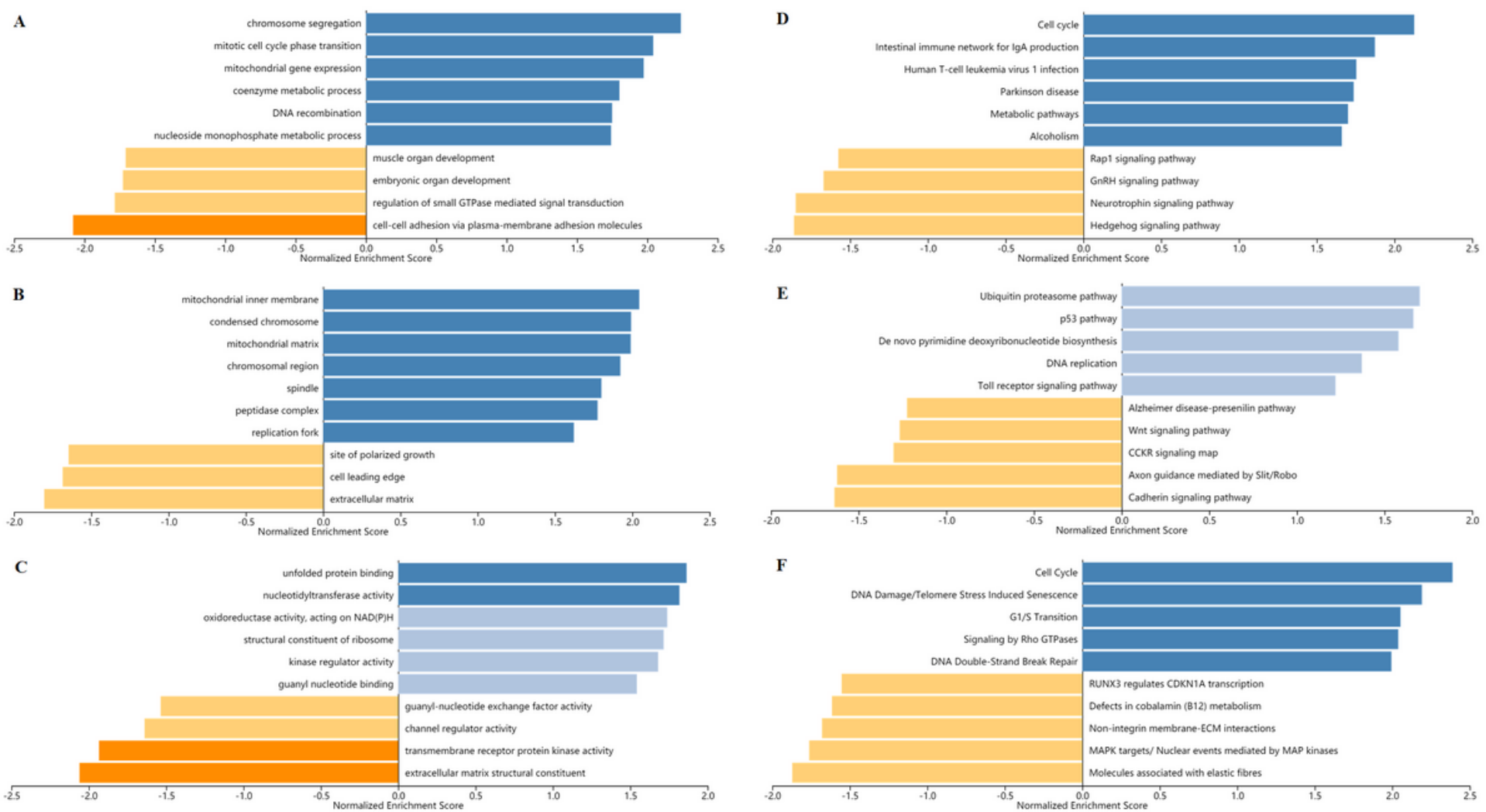

Figure 8

Enrichment analysis of MTHFD2 functional networks in ovarian cancer. A: Biological process. B: Cellular component. C: Molecular function. D: KEGG pathway. E: PANTHER pathway. F: Reactome pathway. 


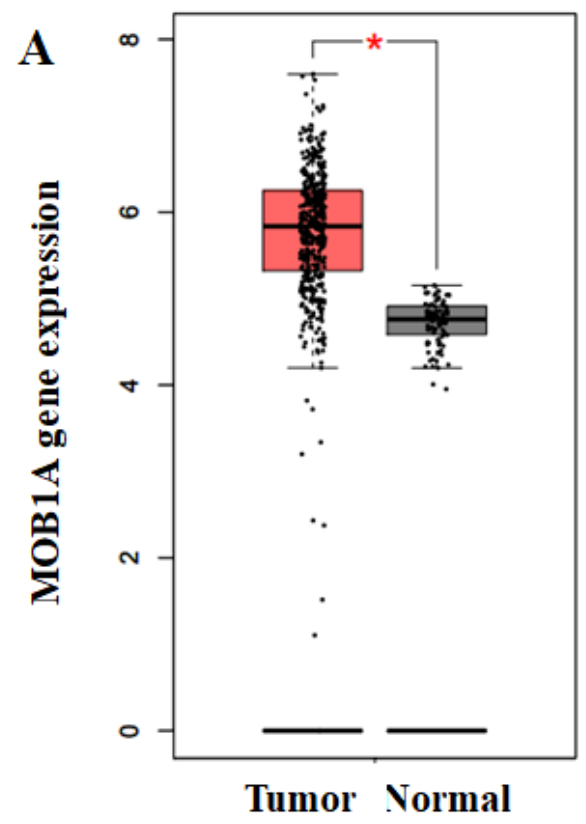

$\operatorname{Num}(T)=426$;

$\operatorname{Num}(\mathbf{N})=88$

Log2FC Cutoff: 1

P-value Cutoff: 0.01

B
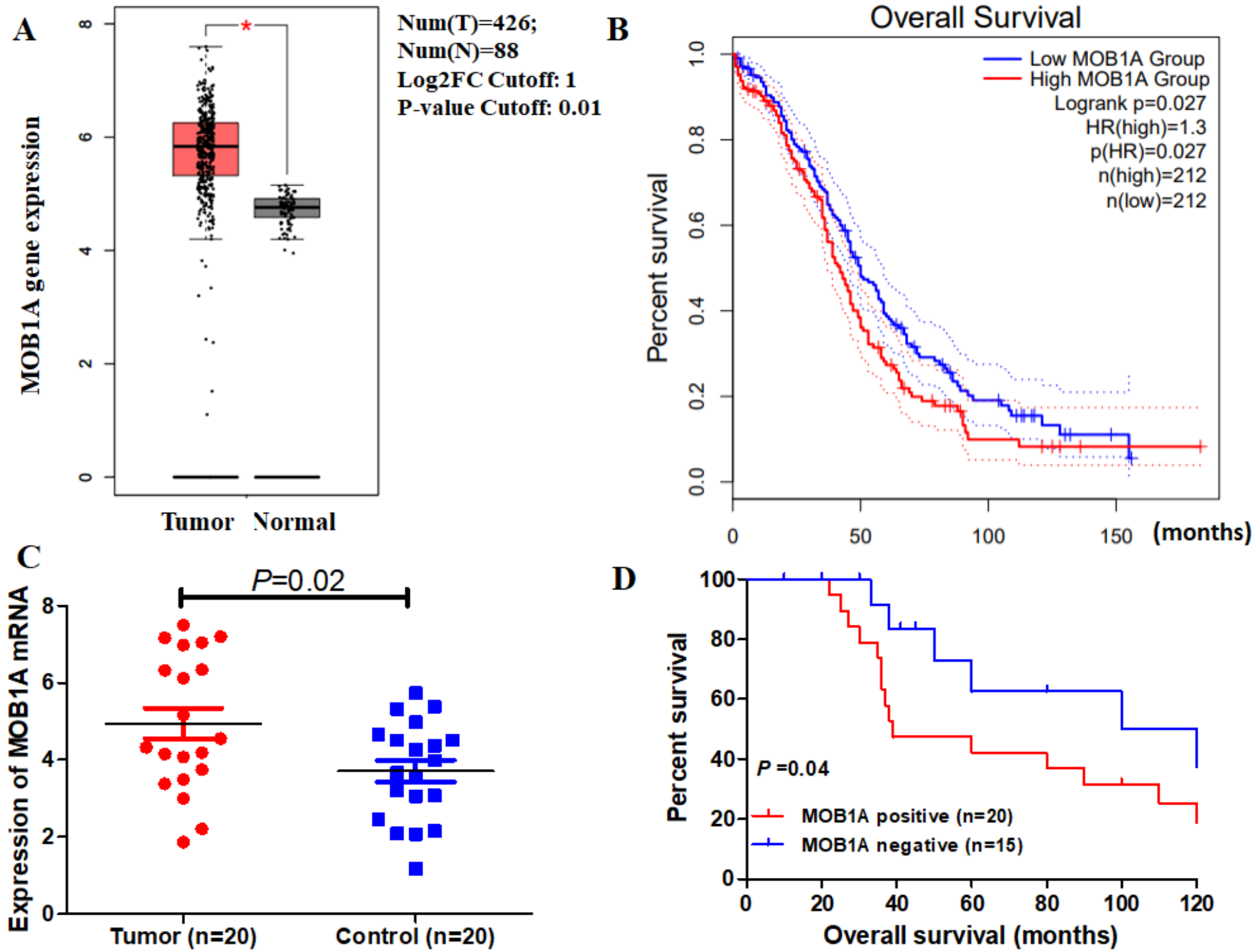

D

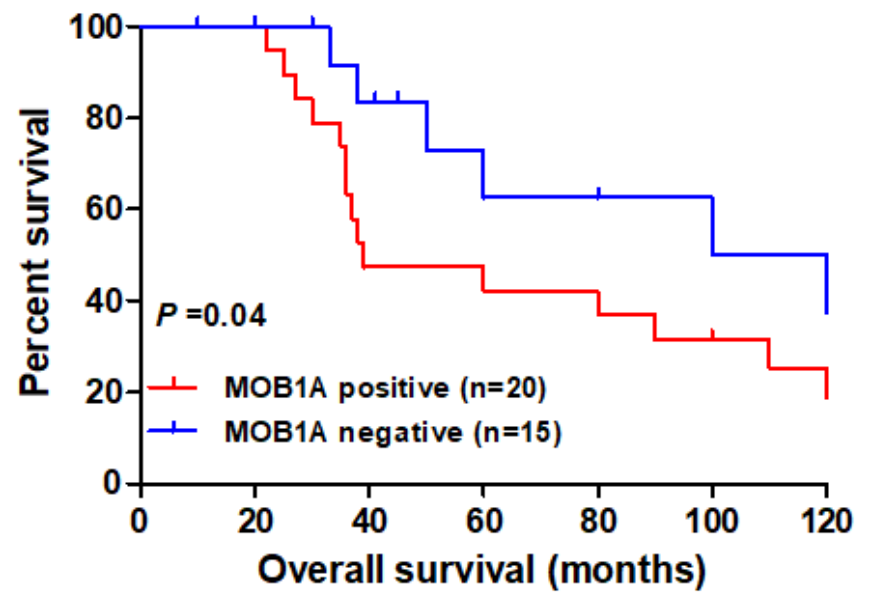

Figure 9

Expression of MTHFD2 in ovarian cancer. A: The expression of MOB1A mRNA in ovarian cancer tissues (red box) and paired normal tissues (black box) from GEPIA. B: Overall survival (OS) curves calculated by PrognoScan database. C: Expression of MOB1A in tumor (20 cases) and adjacent normal tissues (20 cases). D: Kaplan-Meier curves based on MOB1A expression were drawn for overall survival in 35 patients. 

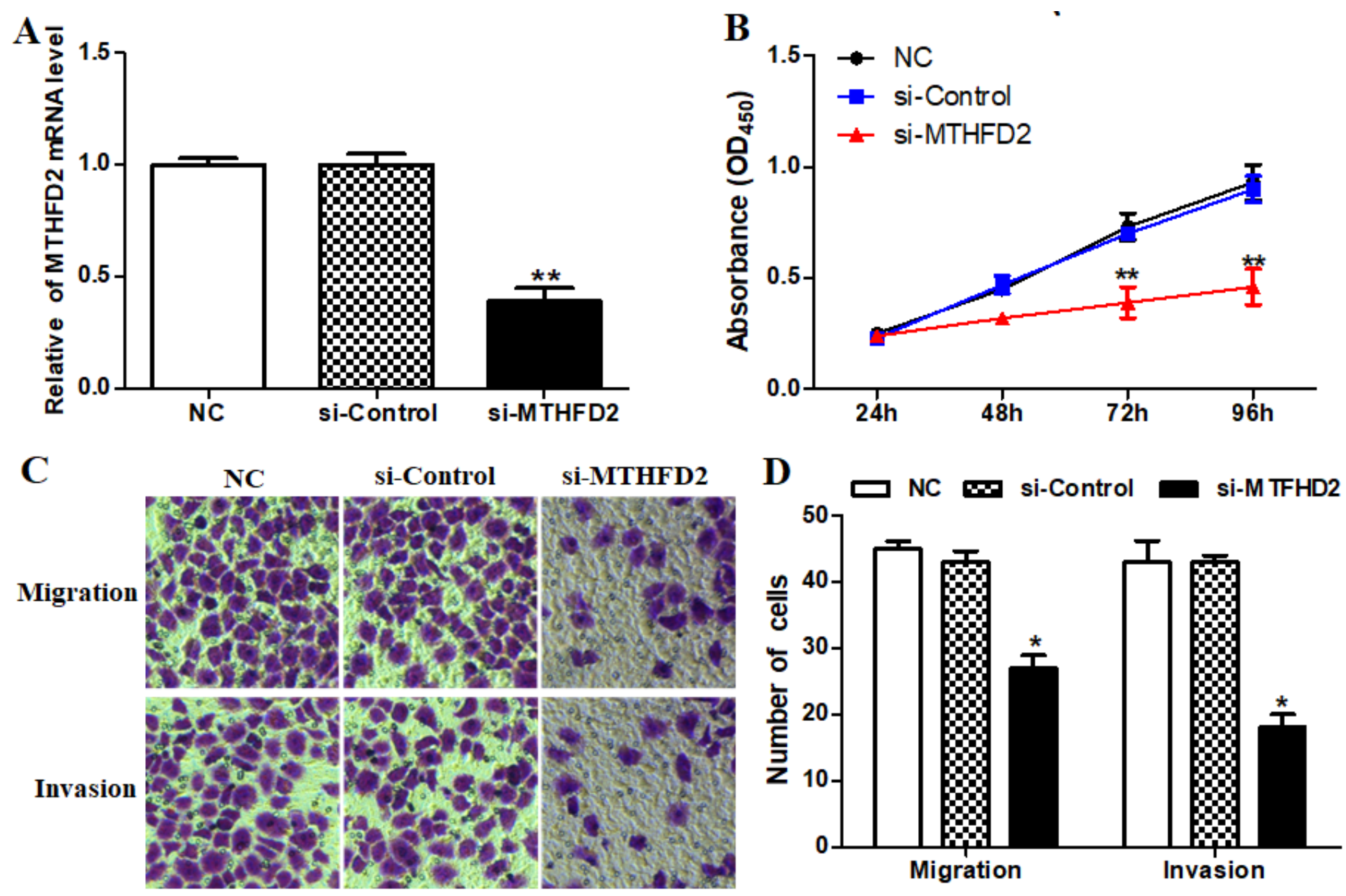

$\mathbf{E}$
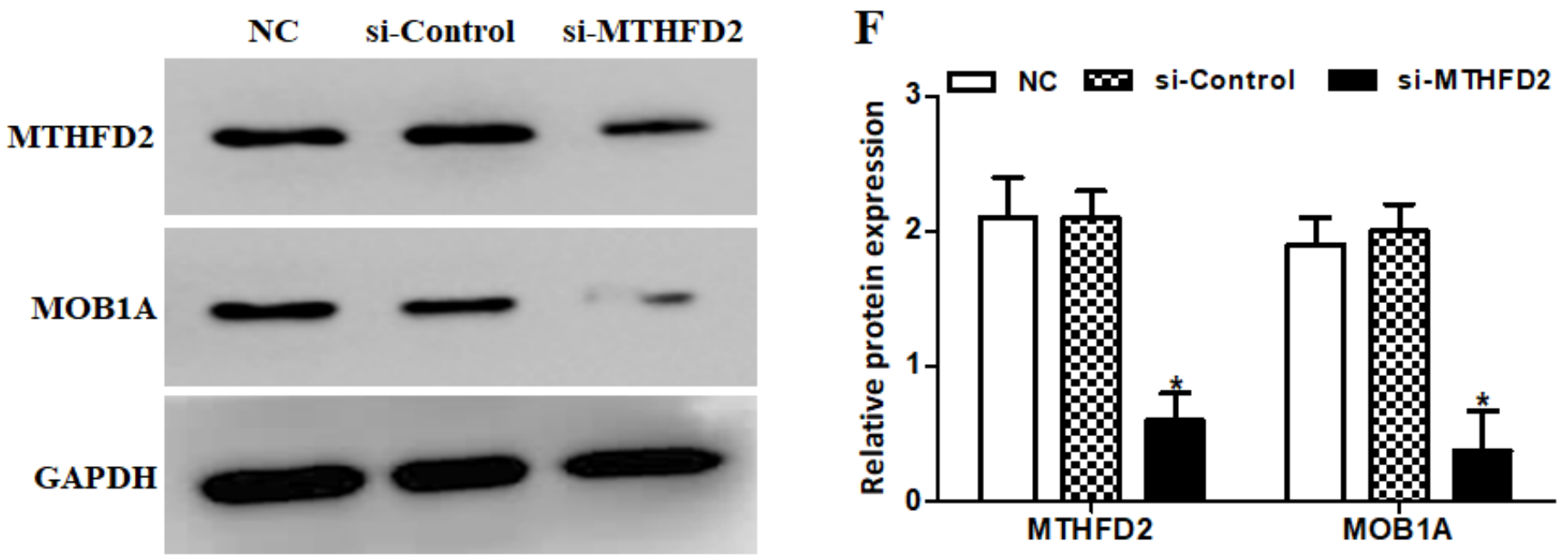

Figure 10

MTHFD2 accelerated the cell growth, invasion, and migration in ovarian cancer through MOB1A. A: The expression of MTHFD2 was significantly reduced in ovarian cancer cells transfected with si-MTHFD2. B: The cell proliferation of ovarian cancer cell lines transfected with different interference sequences, cell proliferation was the weakest in si-MTHFD2 group. C and D: Invasion and migration assays of ovarian cancer cells under different interference conditions. $\mathrm{E}$ and $\mathrm{F}$ : The western blot revealed MOB1A 
expression was significantly decreased in si-MTHFD2 group ovarian cells. ${ }^{*} P<0.05$ and ${ }^{*} P<<0.01$, compared with NC group

\section{Supplementary Files}

This is a list of supplementary files associated with this preprint. Click to download.

- Supplement1.tif 